\title{
PETROLOGÍA Y CRISTALIZACIÓN FRACCIONADA DE LOS BASALTOS - ANDESITAS BASALTICAS DE LA FORMACIÓN GUANEROS (LA YARADA - TACNA - PERÚ)
}

\section{PETROLOGY AND FRACTIONATION CRISTALLISATION THE BASALTS - BASALTICS ANDESITIC THE FORMATION GUANEROS LA YARADA-TACNA- PERU}

\author{
Malpica Sandoval Carlos ${ }^{a}$, Sánchez Izquierdo José ${ }^{b}$, Qispesivana Quispe Luis ${ }^{b}$, Jhon Cervantes ${ }^{b}$
}

\section{RESUMEN}

Las rocas pertenecen a la Formación Guaneros expuestas en el área La Yarada - Tacna - Perú.

Se determinaron dos series volcánicas la inferior (muestras Ly 6, 5, 1, a) y la superior (muestras Ly 12, 14, c, b) constituidas por basaltos y andesitas basálticas. Los espectros de los basaltos de las dos series son semejantes hasta el Erbio, esto indicaría una transición entre el final de la fusión del anfíbol y comienzo de fusión del granate. El proceso de cristalización fraccionada, fusión parcial, cambios químicos del magma y la formación de la textura porfirítica.

Palabra clave: Diagrama de fases, soluciones sólidas, cristalización en equilibrio, fusión parcial, cristalización fraccionada.

\begin{abstract}
The racks concerni un the formation Guaneros exposed in The Yarada -Tacna - Peru (Fig. 1). Si determination two series volcanic the lower (samples Ly 6, 5, 1, a) and the upper (samples Ly 12, 14, c, b), constitutes for basalts and basaltic andesitic. The espectrum of earth rare of the basalts of the two series similar even the erbium, indication a trancition between the end of melting anfibole and begin of the melting garnet. The process fractionation cristallisation, partial melting, change chemical magma and the formation of the texture porfiritic.
\end{abstract}

Key words: Phase diagram, solid solutions, equilibrium crystallisation, partial melting, cristallisation fractionation

\section{INTRODUCCIÓN}

Las muestras se tomaron en el área que pertenece a la La Yarada Tacna-Perú (Cervantes Jhon). La secuencia de los estratos volcánicos en la serie inferior de la base hacia la parte superior es Ly06, Ly05, Ly01 y Lya, en la serie superior la secuencia de los estratos volcánicos de la base hacia la parte superior es Ly12, Ly14, Lyc y Lyb.

\section{OBJETIVOS}

Analizar el origen del magma, la cristalización fraccionada y los cambios químicos que sufren las lavas en el proceso de consolidación de la roca en el sur del Perú.

\section{HIPOTESIS}

Nosotros tenemos dos hipótesis: La primera es que la Ihearzolita de granate fue parcialmente alterada por anfíbol o en su composición tenia anfíbol y al fundirse origino el magma de la serie volcánica inferior, se agota el anfíbol y comienza a fundirse el granate originando el magma para la serie volcánica superior. La segunda hipótesis es que la serie volcánica superior tenga su origen en otro reservorio.
MATERIALES Y METODOS

\section{ESTUDIOS PETROGRAFICOS Y GEOQUIMICOS}

\section{PETROGRAFÍA}

Muestra de la serie volcánica inferior (ly 6, 5, 1, 11a)

Son basaltos y andesitas basálticas constituidas por fenos de plagioclasas alterados por sericita - calcita, corroídos por vidrio, olivinos subhedrales, con una matriz de plagioclasas, piroxenos microgranular a criptocristalina y algunas con vidrio.

Muestras de la serie volcánica superior (ly 12,14, c, b)

Son basaltos y andesitas basálticas con fenos de plagioclasas alterados por sericita clorita y corroídos por vidrio, fenos de piroxenos subhedrales alterados por cloritas, olivino alterado por serpentina. Matriz microgranular con diminutos cristales de plagioclasas, piroxenos, opacos diseminados y vidrio.

\section{GEOQUÍMICA}

Composición de minerales normativos

\footnotetext{
a Universidad Nacional de Cajamarca,

${ }^{\mathrm{b}}$ INGEMME
} 
De acuerdo a la norma CPIW (Tabla 1).

\begin{tabular}{|l|l|l|l|l|l|}
\hline Muestra & Ly 01 & Ly 05 & Ly 11a & Ly 11b & Ly 11c \\
\hline $\mathrm{Cz}$ & 0.00 & 4.17 & 6.74 & 2.81 & 0.79 \\
\hline $\mathrm{Or}$ & 6.06 & 6.83 & 8.45 & 7.78 & 5.50 \\
\hline $\mathrm{Ab}$ & 33.27 & 29.20 & 33.90 & 29.81 & 29.76 \\
\hline $\mathrm{An}$ & 30.74 & 33.08 & 30.67 & 29.63 & 31.94 \\
\hline $\mathrm{Di}$ & 5.49 & 12.47 & 0.00 & 8.31 & 7.77 \\
\hline $\mathrm{Ol}$ & 21.38 & 10.74 & 14.80 & 18.65 & 19.62 \\
\hline $\mathrm{mt}$ & 1.74 & 1.46 & 1.46 & 1.59 & 1.60 \\
\hline $\mathrm{II}$ & 1.77 & 1.84 & 1.93 & 1.52 & 1.82 \\
\hline $\mathrm{C}$ & 0.00 & 0.00 & 1.16 & 0.00 & 0.00 \\
\hline $\mathrm{N}^{\circ} \mathrm{Mg}$ & 0.59 & 0.52 & 0.52 & 0.63 & 0.66 \\
\hline
\end{tabular}

\section{Elementos trazas}

Valores relativos a las condritas de la serie volcánica inferior tiene modelos paralelos de REE y un ligero enriquecimiento en LREE respecto a HREE, con $(\mathrm{La} / \mathrm{Yb}) \mathrm{n}$ de 2.31, en su fuente de origen no incluye al granate, la serie volcánica superior tiene un moderado enriquecimiento de LREE respecto a HREE con $(\mathrm{La} / \mathrm{Yb}) \mathrm{n}$ de 4.2 y el granate esta en su fuente de origen. Los ratios $(\mathrm{La} / \mathrm{Yb}) \mathrm{n}$ nos indican una corteza delgada. Los espectros de los basaltos de las dos series son semejantes hasta el Erbio después son diferentes lo cual indicaría una zona de transición entre el final de la fusión del anfíbol y comienzo de la fusión del granate. En el diagrama de MORB en las dos series se observa una fuerte anomalía negativa de HFSE (principalmente $\mathrm{Nb}$ y $\mathrm{Ti}$ ), es interpretado como una fase residual (anfíbol) en el sólido residual (Wilson 1994). Los valores de normalización se caracterizan por un enriquecimiento en LILE $(\mathrm{K}, \mathrm{Rb}, \mathrm{Ba}$, Th), con un enriquecimiento tenue de LREE con relación a HFSE. El poco enriquecimiento en elementos incompatibles nos da indicios de que no ha tenido una gran contaminación de la corteza continental.

\section{RELACIONES PETROGENÉTICAS}

\section{Cristalización fraccionada}

Del estudio petrográfico y geoquímico se puede entrever que el proceso principal es la cristalización fraccionada la composición química de los minerales será en base a la norma CIPW.

\section{Cristalización de la plagioclasa}

La figura 2 es de Philpotts A. (1990). Tenemos un sistema binario donde las fases están en equilibrio empezamos con una fase liquida en el punto " $M$ " a $1442^{\circ} \mathrm{C}$, en " $A$ " se forman los cristales de plagioclasa, el descenso de la temperatura es muy lento formando los fenocristales, en "B" a $1370^{\circ} \mathrm{C}$ la plagioclasa esta en equilibrio con el líquido en ese punto hay $37 \%$ de cristales y $63 \%$ de líquido. De " $\mathrm{B}$ " a "C" el enfriamiento es más rápido y el desarrollo de los cristales es muy lento formándose una gran cantidad de núcleos estables originando una textura microgranular a criptocristalina. Después del punto " $\mathrm{C}$ " a $1276^{\circ} \mathrm{C}$ la plagioclasa es libre de abandonar el solidus y seguir enfriando pero hay un enfriamiento violento lo que no le permite cristalizar al líquido y se convierte en vidrio formando una matriz microgranular o criptocristalina con vidrio.

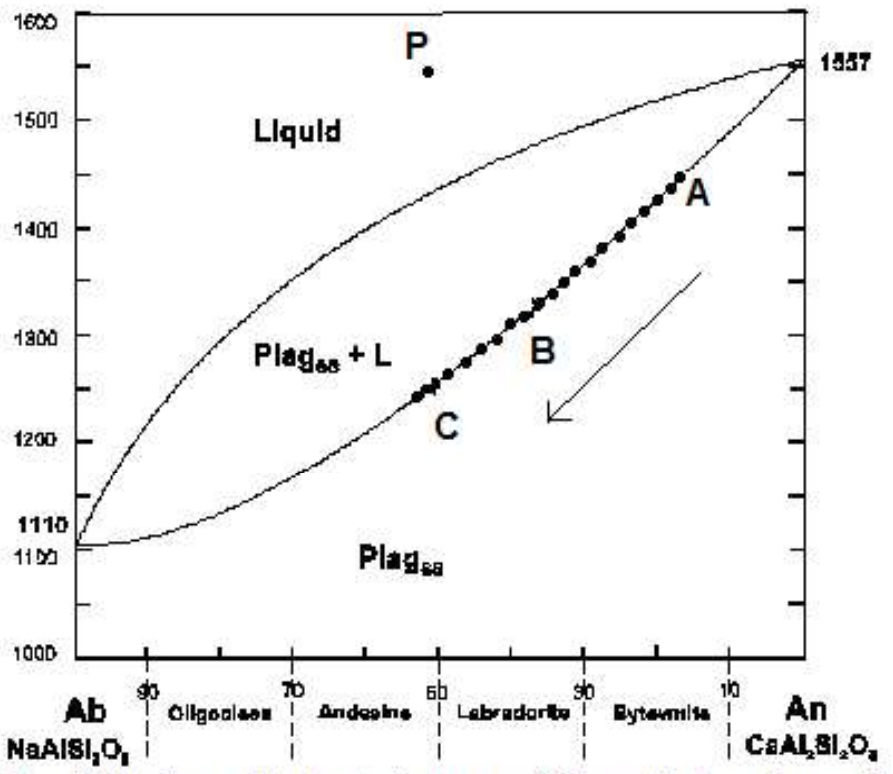

Fig. 2 Solucion solida de plagioclasa se inicia en A y terrrmina en C

\begin{tabular}{|l|l|l|}
\hline Punto & Solución sólida & Líquido \\
\hline $\mathrm{P}$ & & $\mathrm{Ca} 0.49 \mathrm{Na}_{1.01} \mathrm{Al}_{1.49} \mathrm{Si}_{2.51} \mathrm{O}_{8}$ \\
\hline $\mathrm{A}$ & $\mathrm{Ca} 0.8 \mathrm{Na}_{0.4} \mathrm{Al}_{1.8} \mathrm{~S}_{2.2} \mathrm{O}_{8}$ & $\mathrm{Ca}{ }_{0.49} \mathrm{Na}_{1.01} \mathrm{Al}_{1.49} \mathrm{Si}_{2.51} \mathrm{O}_{8}$ \\
\hline $\mathrm{B}$ & $\mathrm{Ca} 0.68 \mathrm{Na}_{0.64} \mathrm{Al}_{1.68} \mathrm{Si}_{2.32} \mathrm{O}_{8}$ & $\mathrm{Ca}_{0.3} \mathrm{Na}_{1.4} \mathrm{Al}_{1.3} \mathrm{Si}_{2.7} \mathrm{O}_{8}$ \\
\hline $\mathrm{C}$ & $\mathrm{Ca} 0.49 \mathrm{Na}_{1.01} \mathrm{Al}_{1.49} \mathrm{Si}_{2.51} \mathrm{O}_{8}$ & \\
\hline
\end{tabular}

\section{Cristalización del olivino}

La figura 3 es de Philpotts A. (1990). Tenemos un sistema binario donde las fases están en equilibrio el líquido en "O", en "A" a $1615^{\circ} \mathrm{C}$ comienza a cristalizar el olivino, el descenso de la temperatura es lento y no sufre cambios bruscos por lo que se forman los fenos de tamaño homogéneo, cuando en " $\mathrm{B}$ " a $1490^{\circ} \mathrm{C}$ hay $50 \%$ de cristales y $50 \%$ de líquido, en " $C$ " a $1392^{\circ} \mathrm{C}$ permanece una sola fase que es el olivino el cual es libre de abandonar el solidus y seguir enfriando.

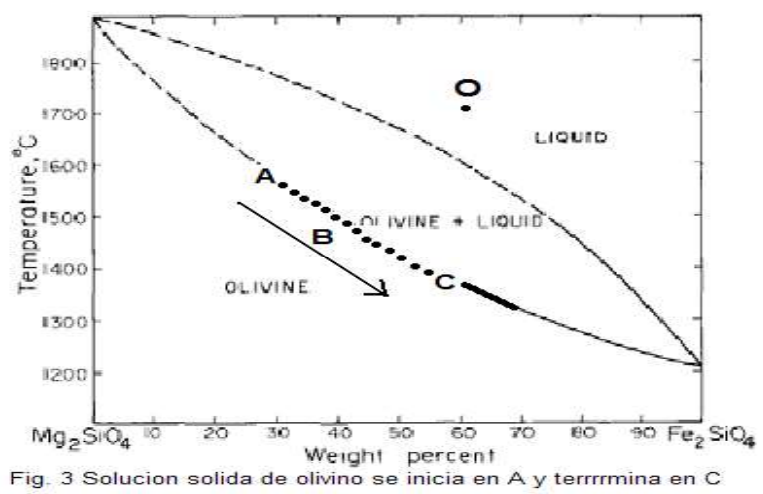

\begin{tabular}{|l|l|l|}
\hline Punto & Solución sólida & Líquido \\
\hline Q & & Mg $_{0.82} \mathrm{Fe}_{1.18} \mathrm{SiO}_{4}$ \\
\hline A & $\mathrm{Mg}_{1.46} \mathrm{Fe}_{0.54} \mathrm{SiO}_{4}$ & $\mathrm{Mg}_{0.82} \mathrm{Fe}_{1.18} \mathrm{SiO}_{4}$ \\
\hline B & $\mathrm{Mg}_{1.18} \mathrm{Fe}_{0.82} \mathrm{SiO}_{4}$ & $\mathrm{Mg}_{0.48} \mathrm{Fe}_{1.52} \mathrm{SiO}_{4}$ \\
\hline C & $\mathrm{Mg}_{0.82} \mathrm{Fe}_{1.18} \mathrm{SiO}_{4}$ & \\
\hline
\end{tabular}




\section{Diagrama de fases diopsido anortita}

La figura 4 de Philpotts A. (1990) En "A" a $1500^{\circ} \mathrm{C}$ comienza la cristalización de anortita y el liquido se enriquece en diopsido, en " $\mathrm{B}$ " a $1392^{\circ} \mathrm{C}$ comienza a formarse los cristales de diopsido por el tamaño de los cristales de diopsido se determino que el enfriamiento es moderado, en el eutectico "E" a $1274^{\circ} \mathrm{C}$ tenemos $63 \%$ de cristales de anortita y diopsido con $35 \%$ de liquido este punto tiene una composición química de $\mathrm{Ca} \mathrm{Mg}_{0.58} \mathrm{Al}_{0.84}$ $\mathrm{Si}_{2} \mathrm{O}_{6.84}$ del cual cristalizan juntos la anortita y el diopsido.

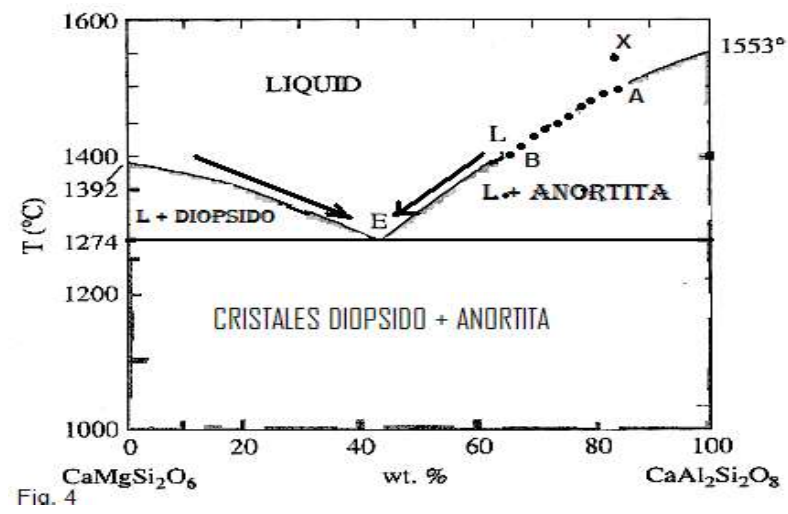

Evolución de los óxidos acorde a la cristalización fraccionada para la muestra ly $11 c$

\begin{tabular}{|l|l|l|l|l|l|l|l|}
\hline & $\begin{array}{l}\text { Wt\% } \\
\text { Roca }\end{array}$ & $\begin{array}{l}\text { sin } \\
\text { olivino }\end{array}$ & $\begin{array}{l}\text { sin olivi. } \\
\text { Cpx }\end{array}$ & $\begin{array}{l}\text { Sin Oli, } \\
\text { Cpx, Plg. }\end{array}$ & $\begin{array}{l}\text { Sin Oli, } \\
\text { Cpx, Plg, } \\
\text { Ort }\end{array}$ & $\begin{array}{l}\text { sin Ol, Cpx } \\
\text { Fpt, Mt-Ilm }\end{array}$ & $\begin{array}{l}\text { todo } \\
\text { cristalizo }\end{array}$ \\
\hline $\mathrm{SiO} 2$ & 50.94 & 53.82 & 53.89 & 44.09 & 16.75 & 100 & 0.00 \\
\hline $\mathrm{Al2O} 3$ & 18.52 & 23.35 & 25.89 & 10.47 & 0.00 & 0.00 & 0.00 \\
\hline $\mathrm{TiO} 2$ & 0.96 & 1.21 & 1.34 & 9.96 & 23.31 & 0.00 & 0.00 \\
\hline $\mathrm{FeO}$ & 7.65 & 2.64 & 1.89 & 14.11 & 33.02 & 0.00 & 0.00 \\
\hline $\mathrm{Fe} 2 \mathrm{O} 3$ & 1.10 & 1.39 & 1.54 & 11.41 & 26.71 & 0.00 & 0.00 \\
\hline $\mathrm{CaO}$ & 8.37 & 10.55 & 9.00 & 0.00 & 0.00 & 0.00 & 0.00 \\
\hline $\mathrm{MgO}$ & 8.26 & 1.21 & 0.00 & 0.00 & 0.00 & 0.00 & 0.00 \\
\hline $\mathrm{Na2O}$ & 3.52 & 4.44 & 4.92 & 0.00 & 0.00 & 0.00 & 0.00 \\
\hline $\mathrm{K} 2 \mathrm{O}$ & 0.94 & 1.18 & 1.31 & 9.75 & 0.00 & 0.00 & 0.00 \\
\hline
\end{tabular}

\section{NATURALEZA DEL ORIGEN}

De Naoki Onuma et al., (1988), determinaron que sus magmas se originaron por fusión parcial del $7 \%$ del manto y por cristalización fraccionada dentro del manto, también por contaminación y cristalización fraccionada dentro de la corteza continental. Su génesis se desarrollo en tres procesos.

1.- Generación de magmas primarios (tipo basalto alto en aluminio) en Ihearzolita de granate.

2.- Cristalización fraccionada del olivino y clinopiroxeno a una profundidad de $30-70 \mathrm{Km}$.

3.- cristalización fraccionada de plagioclasa, clinopiroxeno, ortopiroxeno, hornblenda, biotita y óxidos de hierro a una profundidad somera $(<30 \mathrm{Km})$.

En nuestro caso la Ihearzolita de granate se ha transformado en hornblenda, o tenia hornblenda en su composición original, asumiremos la fusión parcial del $7 \%$ y la cristalización fraccionada es de olivino - clinopiroxeno - plagioclasa - magnetita, IImenita la cual estaría en una etapa intermedia entre los procesos 2 y 3 ; de las muestras Ly c, b, podemos deducir que las rocas menos diferenciadas tienen una composición de olivino y clinopiroxeno después su composición es olivinoclinopiroxeno-plagioclasa y llegaran a una composición de clinopiroxenoevolucionados

plagioclasa en los niveles más

\section{GRADO DE FUSIÓN}

Si asumimos el $7 \%$ de fusión de la Ihearzolita de granate (Naoki Onuma et al., 1988) con una composición de \%Ol $=60, \%$ Cpx $=10$ y \%Piropo $=30$ (Benito García 1994) se puede producir $30 \%$ como máximo de liquido eutectico (Wilson,1989), el numero de magnesio es elevado y las rocas son basaltos y andesitas basálticas esto nos indica que el magma sufrió muy poca diferenciación asumimos que el liquido que origino las rocas es eutectico

Cuando el manto llegue a la temperatura de $1670^{\circ} \mathrm{C}$ en el punto " $E$ " tiene una composición mineralógica de $4.7 \%$ olivino, $48.4 \%$ diopsido y $46.9 \%$ piropo que al fundirse forma un liquido de composición $\mathrm{Mg}_{1.985} \mathrm{Ca}_{0.484} \mathrm{Al}_{0.938}$ $\begin{array}{lll}\mathrm{Si}_{2.422} & \mathrm{O}_{8.72} & \text { en este punto coexisten cuatro fases, el }\end{array}$ mayor aportarte es el diopsido seguido del piropo siendo el olivino el que menos aporta, este liquido es el que migra del manto para almacenarse en la cámara magmática después salir a la superficie y formar posteriormente los basaltos y andesitas basálticas.

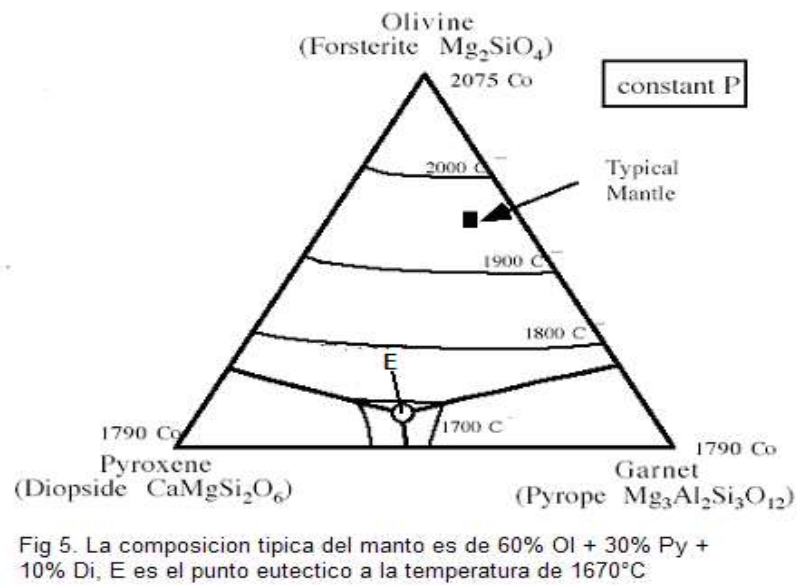

\section{CONCLUSIONES}

1.- Las dos series de rocas de la Formación Guaneros expuesto en el área La Yarada - Tacna, incluye una serie de lavas calco alcalina

2.- Los diagramas de fases nos demuestran como varia la composición química del fundido al cristalizar los minerales, también que los minerales influyen en la concentración de unos elementos más que en otros.

3.- El magma en la cámara se enfriaba lentamente durante el cual crecían los cristales y forman los fenos después hay un periodo de rápido enfriamiento en el cual los cristales crecen poco y un periodo de enfriamiento violento que forma el vidrio este periodo coincide con la erupción de la lava a la superficie.

4.- La hipótesis que planteamos es que hay una alteración de la Ihearzolita de granate a anfíbol o el anfíbol esta como mineral primario y al agotarse el anfíbol comienza a fundir el granate y la otra hipótesis es que la serie volcánica superior pertenece a otro reservorio.

5.- Para un estudio más detallado es necesario hacer microsonda, e isótopos, 


\section{REFERENCIAS BIBLIOGRAFICAS}

[1]. Benito Garcia R. Lopez Ruiz J. (1994). Modelización geoquímica de los procesos de fusión parcial. Estudios geológicos 50. España, pp 291-308

[2]. Fernandez Santin S. y Hernandes Pacheco A. (1991). Calculos y diagramas geoquimicos. España, pp 340

[3]. Onuma Nooki et al., (1982). Geochemical Investigation of the central Andean volcanic zone souther Perú, pp 170.

[4]. Perchuk L.L. y Kushiro I. (1991). Phisical chemistry of magmas. Springer-Verlag, pp. 341.

[5]. Philpotts Anthony R. (1990). Principles of Igneous and Metamorphic Petrology. Prentice Hall. pp 497

[6]. Wilson Marjorie (1989). Igneous Petrogenesis. Unwin Hyman, London, pp 466.

E-mail: cmalpicas@hotmail.com 